\title{
Anatomy and Thermal History of Laser Self-Focusing Damage Tracks in Glass
}

\author{
Wolfgang Haller and Joseph H. Simmons \\ Institute for Materials Research, National Bureau of Standards, Washington, D.C. 20234
}

March 16, 1972

\begin{abstract}
Laser induced self-focusing damage tracks in glass are analyzed. The damaged regions consist of long cylinders of altered refractive index, containing occasional bubbles and partly surrounded by arrays of small cracks. An analysis of the refractive index change in the subfracture damage tracks and the presence of bubbles leads to estimates of a minimum temperature reached by heating of the glass in the tracks. A calculation of minimum stresses developed during laser-beam heating gives a good account of the orientation of the observed cracks.
\end{abstract}

Key words: Laser damage; glass; self-focusing.

\section{Introduction}

Self-focusing damages caused by laser beams in glasses range from gross fractures ("starbursts" and planar cracks) to lines made up of very small bubbles ("bubble tracks") [1]. ${ }^{1}$ Less conspicuous permanent changes in the beam regions are the "filaments," variously described as bright or dark lines of 0.5 to $2.0 \mu \mathrm{m}$ diameter [2]. These filaments appear as regions of altered refractive index and the discrepancies in their reported dimensions stem from difficulties inherent in the interpretation of microscopic refraction images.

Figure 1 shows a typical damage track produced in a piece of glass. In picture B the objective lens focuses upon the track. In pictures $A$ and $C$ the objective lens focuses $9 \mu \mathrm{m}$ respectively behind and before the damage track. In the upper and lower parts of the picture, bubble tracks can be seen. These are separated by a bubble-free region in the center of the picture. A fracture in the glass appears near the lower bubble track. Pictures A and C show the refraction effect of a transparent, cylindrical region in the glass, which obviously has a different refractive index from the surrounding glass matrix. This region extends over the full length of the track and is the object of our present investigation.

\section{Production of Damage}

A $15 \mathrm{~ns}$ pulse from a nominal $10 \mathrm{MW}$ ruby laser was focused into a polished cube of glass by means of a lens with a focal length of $9 \mathrm{~cm}$. The glass had a com-

${ }^{1}$ Figures in brackets indicate the literature references at the end of this paper. position of approximately 61 percent $\mathrm{SiO}_{2} ; 20$ percent $\mathrm{Al}_{2} \mathrm{O}_{3} ; 14$ percent $\mathrm{Na}_{2} \mathrm{O} ; 4$ percent $\mathrm{Li}_{2} \mathrm{O}$ and 1 percent

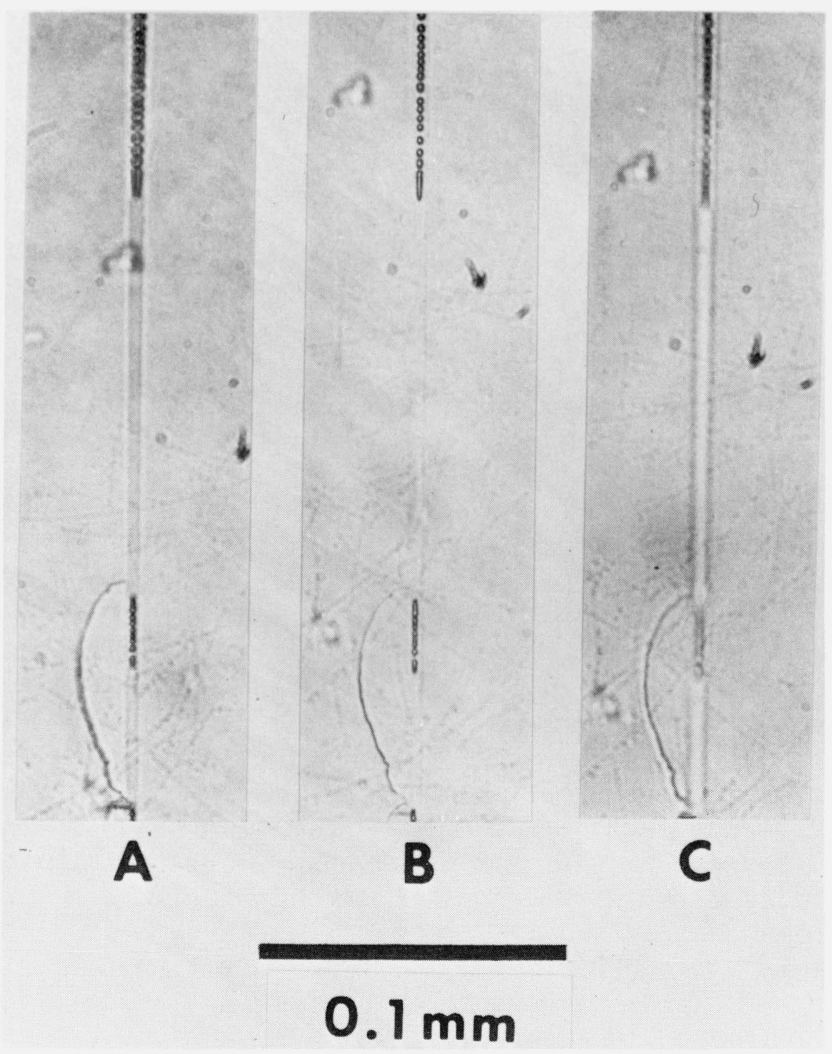

FIGURE 1. Micrographs of a damage track illuminated in transmitted light.

The objective lens is focused $9 \mu \mathrm{m}$ behind (A), on (B), and $9 \mu \mathrm{m}$ before (C) the track. 
$\mathrm{Sb}_{2} \mathrm{O}_{3}$, (weight percent), and a refractive index of 1.516 at $\lambda=0.5467 \mu \mathrm{m}$. A threshold output energy of approximately $0.5 \mathrm{~J}$ was required to produce visible fracture damages in this glass. The laser operated in a single mode by means of a transmission sapphire etalon and had a cryptocyanine-methanol $Q$-switch. A ballistic thermopile-calorimeter was available to monitor the integrated energy of each pulse.

\section{Refractive Index Profile}

The "invisible" subfracture filamentary damage track in the glass specimen was investigated with a micro-Mach-Zehnder twin beam interferometer [3]. The glass sample containing the track was ground and polished to within optical reach of the microscope objective. the reference beam of the interferometer was directed through a simultaneously ground and polished damage-free specimen of the same thickness. Observations were made in the light of the green mercury line $\left(\lambda_{0}=0.546707 \mu \mathrm{m}\right)$. The correction prisms in the reference beam were adjusted until the interference fringes were perpendicular to the track. Photographs were taken on high-speed Polaroid film (3000 ASA) ${ }^{2}$ and these were rephotographed on line film [4] to enhance contrast and eliminate background. Figure 2 shows the interference photomicrograph of the fringes across the track. Some stress birefringence was observed when the track was illuminated with polarized light. The ray polarized parallel to the track appeared to be retarded from the ray polarized perpendicular to the track by an amount corresponding to approximately 30 percent of the total index change. A corresponding difference in peak displacement was observed when parallel and perpendicularly polarized light was used in the Mach-Zehnder interferometer. Since this refractive index anisotropy is a result of the cooling history of the track and is difficult to analyze accurately, we will consider only the average index change in unpolarized light to estimate the minimum temperature which the track reached when heated by the absorption of laser energy during the self-focusing process.

The fringes show a simple displacement as would be produced by a homogeneous cylindrical inclusion of refractive index different from the surrounding matrix. The shape of the displacement gives no evidence of a tubular (hollow) inclusion as has been observed by Davit [2]. The base width of the fringe displacement measures $3 \mu \mathrm{m}$ and it is assumed that this is the diameter of the cylindrical track. On its apex, the fringe displacement measures 0.15 fringe spacings. The direction of the fringe displacement indicates that the inclusion has a lower refractive index than the matrix. The direction of the displacement was tested by observing the movement of the fringes while blowing a gas of high refractive index (freon) through one beam of the microscope. This is in contrast to Davit's observation of an increased index region.

\footnotetext{
${ }^{2}$ Certain commercial materials are identified in this paper in order to specify adequately the experimental procedure. Such identification does not imply recommendation or endorsement by the National Bureau of Standards.
}
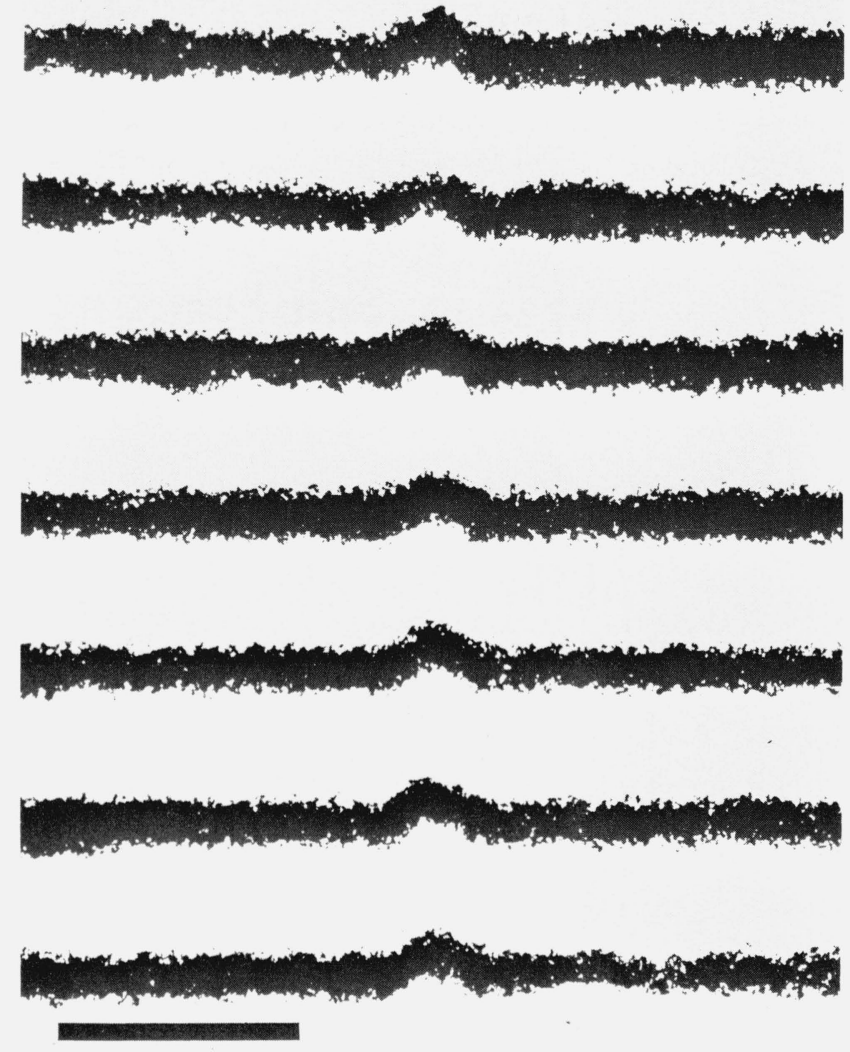

FIGURE 2. Photomicrograph of the damage track taken with a micro-Mach-Zehnder twin beam interferometer at $\lambda=0.54607 \mu \mathrm{m}$.

The black bar shows $10 \mu \mathrm{m}$.

Assuming the track to be a homogeneous cylinder of diameter $d=3 \mu \mathrm{m}$ and refractive index $n_{2}$ embedded in a matrix of refractive index $n_{1}$, allows calculation of the index change in terms of the maximum fractional fringe displacement $f=0.15$ and the wavelength of the light used (in vacuum or air):

$$
n_{1}-n_{2}=\Delta n=\lambda_{0} f / d=2.8 \times 10^{-2} .
$$

The reduced refractive index indicates that the glass has been heated above its transition temperature and has been subsequently cooled rapidly. The arresting of an elevated fictive temperature structure is well known in glasses but under normal quenching conditions it has not produced such large changes. The conditions leading to the reduction in refractive index, $\Delta n$, are discussed later.

The lower refractive index, presumably corresponding to a lower density and to permanent volume expansion in the cylindrical region, has not caused a sharp radial compression in the surrounding matrix. Such a sharp compression would be visible as a counterdeflection of the fringe. The corresponding compression of the surrounding matrix occured apparently over a great distance and the associated fringe shift is therefore not observable. 


\section{Density of Filament}

The reduced refractive index of the cylinder indicates that the glass in the cylinder has a density $\left(\rho_{2}\right)$ different from that of the matrix $\left(\rho_{1}\right)$. The ratio $\rho_{2} / \rho_{1}$ can be estimated from the Lorentz-Lorenz equation:

$$
\frac{\rho_{2}}{\rho_{1}}=\left(n_{1}{ }^{2}+2\right)\left(n_{2}{ }^{2}-1\right) /\left[\left(n_{1}{ }^{2}-1\right)\left(n_{2}{ }^{2}+2\right)\right]
$$

since $n_{1}$, the refractive index of the undamaged glass, is known $\left(n_{1}=1.516\right)$ and $\Delta n$ has been determined interferometrically. $\rho_{2} / \rho_{1}$ is calculated to be 0.95 . In other words, the glass in the track has a density 5 percent below or a specific volume 5 percent above the surrounding matrix. A volume expansion of 5 percent corresponds to a linear expansion of 1.6 percent. This figure is used to calculate the heating stresses in a later part of this paper.

\section{Thermal History of the Filament}

The density and refractive index of an undercooled liquid such as a silicate glass are dependent on its thermal history, i.e., the rate at which the glass is cooled from its molten state. The thermal expansion coefficient is higher for a liquid than a solid. If a melt is cooled rapidly it contracts first as a liquid, but as its viscosity increases, the melt is unable to readjust its structure in pace with the changing equilibrium structure; it solidifies to a glass and then contracts as a solid. In normal technological practice, glasses are cooled at rates of approximately $2-5{ }^{\circ} \mathrm{C} / \mathrm{min}$. At such rates the transition from the liquid to the glassy state occurs near the equilibrium strain-point temperature which is approximately $470^{\circ} \mathrm{C}$ in the glass under investigation. Since the cooling of glasses arrests a hightemperature structure, one speaks of the glass as having a certain fictive temperature. Figure 3 shows idealized curves for a fast and slow cooling treatment of a glass sample.

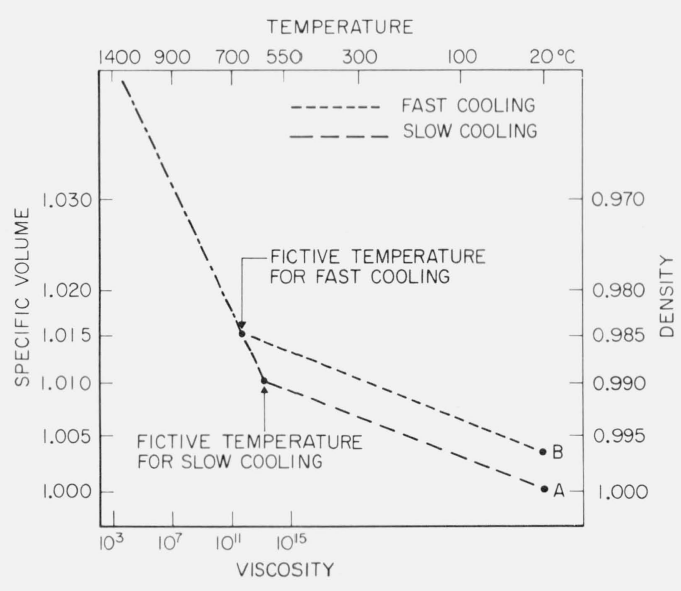

FigURE 3. Idealized cooling curves of a glass.

A and B indicate room temperature densities of slowly and quickly cooled glasses respectively.
The ability of a liquid to adjust its structure with temperature changes is determined by its volume relaxation time. At high temperatures and low viscosities the relaxation times are short and a very rapid cooling rate is required to freeze-in very high temperature structures. Consequently, in order to obtain a certain fictive-temperature structure, two conditions are necessary: (a) the glass must have been heated to at least this fictive temperature and (b) it must have been cooled at a rate sufficiently fast to prevent rearrangement of the structure when cooling below this temperature.

The index change in the damage track allows us to estimate its fictive temperature from the knowledge of the thermal expansion coefficients of the glass. We also can calculate the instantaneous cooling rates of the filament as a function of temperature, compare this rate with the relaxation times at these temperatures, and estimate the temperature $\left(T_{f}\right)$ at which structural rearrangements are arrested. Thus, we have two independent means of estimating the fictive temperature. The only information to be gained by this, however, is the estimate of a minimum temperature (fictive temperature) which the track reached. Much higher temperatures may have been reached during the self-focusing process, but, for the reasons given above, we only have a record of the fictive temperature.

Details of the calculation of $T_{f}$ are shown in Parts 1 and 2 of the Appendix. We find from the fossil refractive index $n_{2}$ and the corresponding density ratio, $\rho_{2} / \rho_{1}$, that the track has at least experienced a temperature of 1450 to $2800^{\circ} \mathrm{C}$. (The range is caused by uncertainty in estimating a value for the high temperature expansion coefficient.) In all probability the temperature reached was much higher. Since, however, the cooling rate of the filament is $10^{10}{ }^{\circ} \mathrm{C} / \mathrm{s}$ (see Part 2a of Appendix) a fictive temperature not higher than 1600 to $1800{ }^{\circ} \mathrm{C}$ can be frozen in. At this temperature the relaxation time becomes so long (see Part $2 \mathrm{~b}$ of the Appendix) that this high-temperature structure becomes arrested.

An additional clue is provided by the observation of microscopic bubbles in the track which indicates a minimum estimate of $1500^{\circ} \mathrm{C}$. The manufacture of silicate glasses requires that the melt be maintained at approximately $1500{ }^{\circ} \mathrm{C}$ for many hours in order to facilitate the expulsion of dispersed and dissolved gases $\left(\mathrm{N}_{2}, \mathrm{O}_{2}, \mathrm{CO}_{2}, \mathrm{SO}_{2}\right)$. At the elevated temperature, the melt still contains dissolved residual gases in concentrations which correspond to the solution equilibrium at the melting temperature and atmospheric pressure. When such melts are cooled into the glassy state, uptake of more gases is minimal because of the slow diffusion. In order to produce gas bubbles in glass, it is therefore generally necessary to heat it above the temperature at which the melt was equilibrated with the atmosphere. Under the volume-restraining condition of laser track formation, the temperature of bubble formation would, however, be considerably higher than at atmospheric pressure.

All the facts discussed above support the assumption of considerable heating during track formation-at 
least to temperatures at which the glass has a viscosity below $10^{2} \mathrm{P}$, i.e., the viscosity at which bubble formation is expected. This assumption is also supported by evidence from other workers. Harper [5] observed the extrusion of glass fibers from laser fractures. Hercher [1] observed that black body continuum radiation was emitted during the damage and estimated a maximum temperature of $5000 \mathrm{~K}$.

\section{Thermal Stresses}

Using the model of an expanding infinitely long cylinder with a diameter of $3 \mu \mathrm{m}$ embedded in an infinite solid of the same mechanical properties, we have attempted to calculate the stresses in and around the damage filament. In the equations, we assumed that the cylinder expanded by 5 percent of its volume or 1.6 percent of its length isotropically. This is the total expansion indicated by our observation of the cold, fossil track. The expansion of the hot cylinder during the actual laser damage was undoubtedly much higher. We calculate (see Appendix Part 3) the minimum stresses in and around the cylinder produced during heating of the damage area to be as follows:

A. Outside the cylinder at the surface of the track, $r=1.5 \mu \mathrm{m}$, following the convention that tensile stresses are positive, and compressive ones negative, the radial, $\sigma_{r r}$; tangential, $\sigma_{\theta \theta}$; and longitudinal, $\sigma_{z z}$, stresses are listed below:

$$
\begin{aligned}
& \sigma_{r r}=-6.5 \times 10^{8} \mathrm{~N} / \mathrm{m}^{2}=[-95,000 \mathrm{psi}] \\
& \sigma_{\theta \theta}=+6.9 \times 10^{8} \mathrm{~N} / \mathrm{m}^{2}=[+100,000 \mathrm{psi}] \\
& \sigma_{z z}=+0.46 \times 10^{8} \mathrm{~N} / \mathrm{m}^{2}=[+6,800 \mathrm{psi}] .
\end{aligned}
$$

B. Inside the cylinder, also at the surface, $r=1.5 \mu \mathrm{m}$ :

$$
\begin{aligned}
& \sigma_{r r}^{\prime}=-6.5 \times 10^{8} \mathrm{~N} / \mathrm{m}^{2}=[-95,000 \mathrm{psi}] \\
& \sigma_{\theta \theta}^{\prime}=-6.5 \times 10^{8} \mathrm{~N} / \mathrm{m}^{2}=[-95,000 \mathrm{psi}] \\
& \sigma_{z z}^{\prime}=-12.8 \times 10^{8} \mathrm{~N} / \mathrm{m}^{2}=[-190,000 \mathrm{psi}] .
\end{aligned}
$$

The tangential stress in the matrix is tensile, and has the highest magnitude. Thus, it would preferentially lead to a fracture. Its actual numerical value, even considering the minimum volume change, is more than $\frac{1}{5}$ of the stress considered to be the inherent, internal strength of glass in absence of defects [6]. In the presence of the usual defects and considering the higher hot stresses around the track, the tangential stresses would be sufficient to initiate cracks. Such cracks would be expected to occur in planes perpendicular to the tangential stress direction, and thus would be in half-planes parallel to and containing the axis of the track, randomly distributed around the circumference.

The microscopic study of the numerous tracks produced in the glass, as described earlier, reveals two types of fractures: (a) gross starburst, usually not more than one or two per track and (b) very numerous, small planar circular fractures along all tracks, associated both with bubble regions of the track, and with the transparent track region between the bubbles.

All the planar fractures are almost circular, rest with one edge against the track and are in planes containing the axis of the track. Figure 4 is a sketch of the described fractures. We did not find any fractures perpendicular to the track as had been described for laser damage in polymethyl-methacrylate [7]. We believe that the gross starburst cracks were caused by foreign inclusions, while the small ubiquitous planar fractures are associated with an intrinsic self-focusing damage process.

The morphology of the observed planar fractures is in agreement with the findings of the stress calculation which predict the dominance of tangential stresses.

\section{Summary}

(1) Laser induced self-focusing damage tracks in glass were produced by passive irradiation.

(2) The refractive index distribution in the damaged glass was investigated by double-beam interferometry.

(3) Filamentary damages were found which consist of homogeneous cylindrical regions, $3 \mu \mathrm{m}$ in diameter, having a refractive index of $\Delta n=2.8 \times 10^{-2}$ below the surrounding glass.

(4) The reduced refractive index in the damaged region is calculated to correspond to a reduction in density by a factor of $\rho_{2} / \rho_{1}=0.95$.

(5) It is calculated that the glass in the filament has a structure corresponding to a fictive temperature of 1450 to $2800^{\circ} \mathrm{C}$, the range being caused by uncertainty in the value of the high temperature expansion coefficient.

(6) It is calculated from heat flow conditions and a typical relaxation spectrum of glass that it is not possible to freeze-in a high-temperature structure corresponding to a higher fictive temperature than 1600 to $1800^{\circ} \mathrm{C}$, even though the actual temperature during laser beam exposure may have considerably exceeded this temperature.

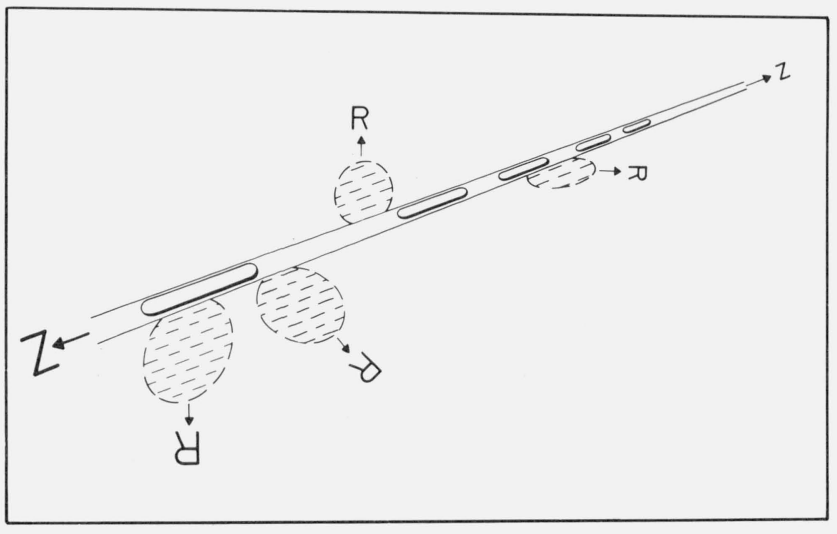

FIGURE 4. Sketch of the small planar fractures observed along all tracks.

The fractures are in half-planes containing the track axis $(z)$ and a random radial vector $\mathbf{R}$. 
(7) Bubble formation in damage tracks is evidence that the laser beam heated the glass at least to $1500^{\circ} \mathrm{C}$.

(8) Equations for the axial, radial and tangential stresses in and around a heated cylindrical region in glass are derived. It is shown that for heating the cylinder to its fictive temperature only, corresponding to a volume change of 5 percent, the tensile tangential stress reaches as much as 100,000 psi which is $\frac{1}{5}$ of the inherent, internal strength of glass in absence of defects.

(9) A microscopic investigation of the laser tracks shows large numbers of small planar fractures originating from one side of the track and oriented parallel to the track. Such fractures have the proper orientation to be caused by the predicted dominant tangential stresses arising from laser-beam heating.

(10) It is concluded that thermal expansion of the glass is a sufficient mechanism to produce fractures within the glass. This conclusion is supported by the fact that glasses with low thermal expansion (fused silica, borosilicate glasses) show a higher damage threshold to passive laser beam exposure than glasses with high thermal expansion [8].

\section{Appendix}

Parts I and II of this appendix show detailed calculations of the estimated ranges within which the fictive temperature of the damage track occurs. These calculations rest upon assumptions and equations which are well followed, in general, by silicate glasses, and are only proposed to give an estimate of the minimum temperature reached by the damage track. Each part presents totally independent analyses which rest on different assumptions and available data.

Part III is a rigorous analysis of the stresses involved in the heating process and does not depend upon the results of Parts I and II. Since we are calculating stresses developed in heating, and the heating process was very fast, we may neglect the effect of plastic deformations, or volume relaxations and compaction effects. Therefore, Part III is only concerned with the elastic problem of heating a cylindrical filament within a cold matrix.

\section{Part I. Calculation of Fictive Temperature from Index Change}

The estimate of a fictive temperature from a change in index depends upon the variation of index with specific volume, and of specific volume with temperature. From the Lorentz-Lorenz equation, we can easily estimate $\Delta v$ and $\Delta n$, but the dependence of specific volume on temperature up to high temperatures is only known in general terms. We know the expansion coefficient of this glass up to $300^{\circ} \mathrm{C}$ and may apply it as high as the transformation range $\left(550^{\circ} \mathrm{C}\right)$. We also have measured the temperature dependence of index in the transformation region from 550 to $580^{\circ} \mathrm{C}$. From these data, we may calculate an upper and a lower temperature estimate for $T_{f}$, as shown in figure 5 . The relative size is plotted versus temperature

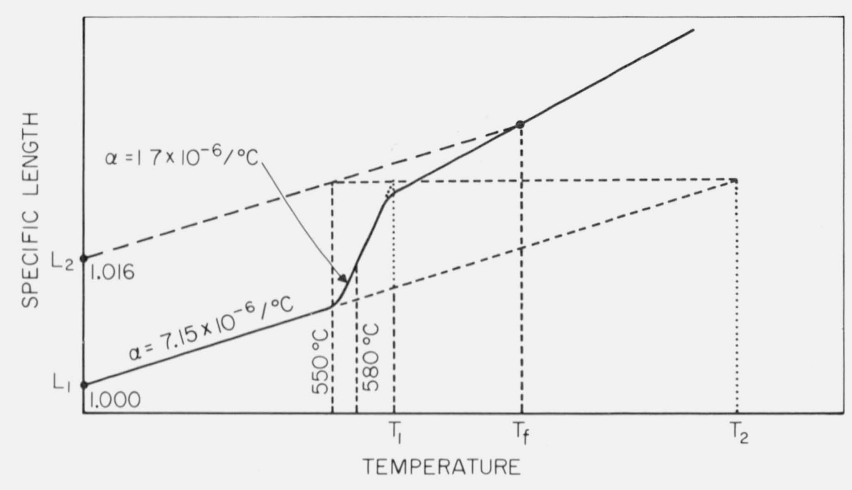

FIGURE 5. Extrapolation procedure for estimating the high temperature specific volume of the glass.

$T_{1}$ and $T_{2}$ are the upper and lower estimates of the fictive temperature. The measured solid expansion coefficient is shown. The expansion coefficient quoted for the transformation range $\left(550-580^{\circ} \mathrm{C}\right)$ has been calculated from the index data by using the LorentzLorenz equation, and is only shown here for illustration.

and follows the general behavior expected of silicate glasses. The estimate, $T_{1}$, is an extrapolation of the index dependence on temperature. It uses the expansion coefficient characteristic of the transformation region, and will underestimate $T_{f}$. The estimate $T_{2}$ uses the solid expansion coefficient and will overestimate $T_{f}$.

Measurements of index change as a function of temperature in the transformation range from 550 to $580^{\circ} \mathrm{C}$ are shown below:

\begin{tabular}{c|c}
\multicolumn{2}{c}{ TABLE 1} \\
\hline \hline$n(\lambda=0.58959 \mu \mathrm{m})$ & Temperature $^{\circ}$ \\
\hline 1.51337 & $584^{\circ} \mathrm{C}$ \\
1.51363 & $575^{\circ} \mathrm{C}$ \\
1.51406 & $562^{\circ} \mathrm{C}$ \\
1.51436 & $552^{\circ} \mathrm{C}$ \\
\hline
\end{tabular}

These points plot as a straight line whose slope yields the temperature dependence of index:

$$
\left[\frac{\Delta n}{\Delta T}\right]=-3.16 \times 10^{-5} /{ }^{\circ} \mathrm{C}
$$

The observed change of $\Delta n=-0.028$ gives a temperature change of $900^{\circ} \mathrm{C}$. The base glass is assumed to have a fictive temperature of $550{ }^{\circ} \mathrm{C}$, therefore we estimate $T_{1} \simeq 1450^{\circ} \mathrm{C}$.

In the calculation of $T_{2}$, we now invoke the LorentzLorenz equation to calculate the difference in volume between the track and matrix. If $n_{1}$ is the index of the matrix at 1.516, corresponding to a matrix fictive temperature of $550^{\circ} \mathrm{C}$, and $\Delta n$ is -0.028 we may calculate $n_{2}$ to be 1.488 and calculate the size ratio $L_{2} / L_{1}$ to be 1.016 . The solid expansion coefficient is $7.15 \times 10^{-6} /{ }^{\circ} \mathrm{C}$, therefore, the corresponding temperature change is calculated to be $2240^{\circ} \mathrm{C}$. Adding the fictive temperature of the matrix leads to an estimate for $T_{2} \simeq 2800^{\circ} \mathrm{C}$. The fictive temperature estimate is therefore seen to be between 1450 and $2800^{\circ} \mathrm{C}$ by this method. 
Part II. A. Calculation of Cooling Rate of Filament

The hot filament may cool by thermal conduction and/or radiation. Due to the good thermal contact assumed between the hot zone and its surroundings, the heat flow by conduction is much larger than by radiation, and the latter may be totally neglected.

The problem of a hot cylinder embedded in a matrix has been solved by Carslaw and Jaeger [9] for the change in temperature of the matrix as a function of time and distance from the hot zone. Due to the good thermal contact between the cylinder and matrix, we expect a very high cooling rate, therefore, we are only interested in the short-time solutions. The cooling rate may be calculated from the heat flow between the hot cylinder and a larger concentric cold sink surface. We shall, therefore, use the solutions of Carslaw and Jaeger to estimate the distance of this cold sink from the hot zone. An analysis of their calculations [fig. 41, p. 337, ref. 9] leads to table 2 below, assuming a heat conductivity, $K=0.0121 \mathrm{~J} \mathrm{~cm}^{-1} \mathrm{~s}^{-1}{ }^{\circ} \mathrm{C}^{-1}$; a specific heat, $C=1.34 \mathrm{~J} \mathrm{~g}^{-1}{ }^{\circ} \mathrm{C}^{-1}$; a density, $\rho=2.1 \mathrm{~g} / \mathrm{cm}^{3}$, and a filament radius $a=1.5 \mu \mathrm{m}$.

TABLE 2

\begin{tabular}{l|c|c}
\hline \hline $\begin{array}{c}\text { Distance from filament } \\
r_{0} / a\end{array}$ & $\begin{array}{c}\text { Temperature at } r_{0}: \\
T_{0} / T \text { (filament) }\end{array}$ & $\begin{array}{c}\text { Time taken to increase } \\
\text { the temperature of } r_{0} \\
\text { to the value } T_{0}\end{array}$ \\
\hline 10 & 0.4 & $8 \times 10^{-2} \mathrm{~s}$ \\
10 & .2 & $8 \times 10^{-3}$ \\
1.4 & .4 & $3.2 \times 10^{-5}$ \\
1.17 & .4 & $2.4 \times 10^{-6}$ \\
1.17 & .2 & $8 \times 10^{-7}$ \\
1.09 extrapolate & .4 & $8 \times 10^{-7}$ \\
1.04 extrapolate & .4 & $2.4 \times 10^{-7}$ \\
\hline
\end{tabular}

Since we are interested in finding the cooling rate applicable for the first $200{ }^{\circ} \mathrm{C}$ drop in temperature, and the cooling rates we expect to obtain are of the order $10^{9}$ to $10^{10}{ }^{\circ} \mathrm{C} / \mathrm{s}$, let us consider a cold sink at $r_{0}=1.17 a$ for a slow rate, and $r_{0}=1.04 a$ for a fast cooling rate estimate. The cooling rate can be calculated as follows:

$$
\frac{d T}{d t}=\frac{1}{\rho V C} \frac{d q}{d t}=\frac{2 \pi a L K\left(T_{\mathrm{fil}}-T_{0}\right)}{C \pi \rho a^{2} L\left(r_{0}-a\right)}=\frac{2 K\left(T_{\mathrm{fil}}-T_{0}\right)}{C \rho a\left(r_{0}-a\right)}
$$

where $K$ is the thermal conductivity, $d q / d t$ is the rate of heat flow, $C$ is the specific heat, $V$ is the filament volume, $L$ is a unit length and $T_{0}$ is the matrix temperature at $r_{0}$. The low and high rates $\left(r_{0}=1.17 a\right.$ and $\left.r_{0}=1.04 a\right)$ are calculated respectively to be:

$$
\begin{aligned}
& \left(\frac{d T}{d t}\right)_{l}=2.3 \times 10^{6}\left[T_{\mathrm{fil}}-T_{0}\right]\left({ }^{\circ} \mathrm{C} / \mathrm{s}\right) \\
& \left(\frac{d T}{d t}\right)_{h}=9.5 \times 10^{6}\left[T_{\mathrm{fil}}-T_{0}\right]\left({ }^{\circ} \mathrm{C} / \mathrm{s}\right) .
\end{aligned}
$$

The resulting heat conduction cooling rates are listed in table 3 below and it is seen that the filament cools fast enough to undergo a temperature change of approximately $100^{\circ} \mathrm{C}$ in less than $4 \times 10^{-8} \mathrm{~s}$, and therefore our assumed cold sinks remain so during that period. In the calculation of cooling rates, it is, therefore, suitable to assume that $T_{0}$ represents room temperature, $20^{\circ} \mathrm{C}$, and thus ignore it.

TABLE 3

\begin{tabular}{c|l|l}
\hline \hline Filament temperature & \multicolumn{1}{|c|}{$\left(\frac{d T}{d t}\right)_{h}$} & $\left(\frac{d T}{d t}\right)_{l}$ \\
\hline $1000^{\circ} \mathrm{C}$ & $1.0 \times 10^{10}{ }^{\circ} \mathrm{C} / \mathrm{s}$ & $2.4 \times 10^{9}{ }^{\circ} \mathrm{C} / \mathrm{s}$ \\
$1200^{\circ} \mathrm{C}$ & 1.2 & 2.8 \\
$1400^{\circ} \mathrm{C}$ & 1.4 & 3.3 \\
$1600^{\circ} \mathrm{C}$ & 1.6 & 3.8 \\
$1800^{\circ} \mathrm{C}$ & 1.8 & 4.2 \\
$2000^{\circ} \mathrm{C}$ & 2.0 & 4.7 \\
$2200^{\circ} \mathrm{C}$ & 2.2 & 5.2 \\
\hline
\end{tabular}

\section{Part II. B. Calculation of Volume Relaxation Time for the Filament}

The volume relaxation time at high temperatures may be estimated by comparing this glass to a sodiumborosilicate for which high-temperature data have been reported [10]. At temperatures above $1400{ }^{\circ} \mathrm{C}$ most oxide glasses are found to have a single or narrow distribution of relaxation times, therefore, characterizing the most probable relaxation time will suffice to analyze the relaxation processes. Results from the sodaborosilicate study [10] also show that at high temperatures the volume relaxation time is approximately 3 times the shear relaxation time. The latter may then be found by considering the shear viscosity data, and comparing it with the soda-borosilicate (SBS) glass [10]:

$$
\tau=\frac{\eta}{\eta_{\mathrm{SBS}}} \tau_{\mathrm{SBS}}
$$

This equation rests upon the likely assumption that the moduli of the two glasses are equal. This falls well within the order of magnitude estimate which we are seeking. The volume relaxation time is thus found as:

$$
\tau_{v}=3 \frac{\eta}{\eta_{\mathrm{SBS}}} \tau_{\mathrm{SBS}}
$$

Once $\tau_{v}$ is calculated, we have the rate at which the structure rearranges itself. The minimum cooling rate necessary for freezing a given structure is found by requiring $\tau_{v}$ to be the time necessary for the relaxation time to increase by a factor of 2. This factor approximates the width of what is usually assumed to be a single relaxation process. Thus, by the time $\tau_{v}$ has elapsed, it would require the structure $2 \tau_{v}$ to rearrange and so on until room temperature is reached. Once the cooling rate necessary to freeze a structure equals the heat conduction cooling rate, then the latter is a likely mechanism for arresting structural changes at the tem- 
perature of coincidence. The volume relaxation time and minimum cooling rates are given in table 4 below:

TABLE 4

\begin{tabular}{c|c|c}
\hline \hline Temperature & Minimum cooling rate & $\begin{array}{c}\text { Volume relaxation } \\
\text { time } \tau_{v}\end{array}$ \\
\hline 1000 & $2.5 \times 10^{6}{ }^{\circ} \mathrm{C} / \mathrm{min}$ & $10^{-5} \mathrm{~s}$ \\
1200 & $10^{8}$ & $5 \times 10^{-7}$ \\
1400 & $10^{9}$ & $3 \times 10^{-8}$ \\
1600 & $8 \times 10^{9}$ & $6 \times 10^{-9}$ \\
1800 & $4 \times 10^{10}$ & $2 \times 10^{-9}$ \\
2000 & $1.5 \times 10^{11}$ & $5 \times 10^{-10}$ \\
2200 & $4 \times 10^{11}$ & $2 \times 10^{-10}$ \\
\hline
\end{tabular}

Referring to table 3 we may now compare the two aforementioned cooling rates, and find the point of coincidence. An upper and a lower estimate of the conduction cooling rate were calculated, therefore, we have two temperature estimates. The temperatures obtained from these calculations give us an estimate of fictive temperature from 1600 to $1800{ }^{\circ} \mathrm{C}$.

\section{Part III. Solution of Stresses Developed by Heating of the Laser Track}

In this section, we are interested in calculating the stresses developed both inside and outside the cylinder representing the laser track when its temperature reaches a value corresponding to the measured change in index. By the Lorentz-Lorenz equation, we can calculate the change in volume associated with the index change without having to speculate about the temperature dependence of the index of this glass. The stresses can then be directly calculated from the volume change.

After a somewhat detailed look through the literature, for a general solution of the problem, we unfortunately found that the most common solutions involve the "plane-strain" approximation. This approach assumes that there is no strain in the longitudinal direction and that the necessary stress is provided externally to the system. The solution does not represent the problem at hand, since a large longitudinal stress is expected to develop in the cold matrix. The need to determine all stresses in the matrix (outside the hot cylinder) requires a more general solution which is given below.

Some solutions of the generalized plane-strain problem may be found in the literature [13], where, in this case, the longitudinal stress is assumed to be a constant. We have not found one which proceeded beyond the first motions of solving the problem, therefore, we have chosen to solve the problem from the general stress-strain relations.

The problem is best expressed in cylindrical coordinates, since the angular symmetry eliminates the $\theta$-dependence of the solutions. The general stressstrain relations used here are taken from Love [11], and particularly from Boley and Weiner [12].

The solutions derived below are based upon the assumption that the positive volume change of the cylinder in the longitudinal $(z)$ direction occuring during the heating process, induces stresses outside the cylinder which occur throughout the cold matrix and reach a maximum at the interface. Outside the cylinder, the longitudinal extension $\left(U_{z}\right)$ is therefore proportional to the length [or distance away from the origin which we locate in the middle of the cylinder], and the longitudinal strain is uniform. The latter, $\epsilon_{z z}$, is only a function of the radial distance:

$$
U_{z}=f(r) z ; \quad \epsilon_{z z}=f(r)
$$

This allows solution of the equilibrium equation in the axial direction:

$$
\frac{\partial \sigma_{r z}}{\partial r}+\frac{\partial \sigma_{z z}}{\partial z}+\frac{\sigma_{r z}}{r}=0
$$

where

and

$$
\sigma_{r z}=\mu\left[\frac{\partial U_{r}}{\partial z}+\frac{\partial U_{z}}{\partial r}\right]
$$

$$
\sigma_{z z}=(\lambda+2 \mu) \epsilon_{z z}+\lambda \epsilon_{r r}+\lambda \epsilon_{\theta \theta}
$$

$\lambda$ and $\mu$ are the Lamé elastic constants for the material, and the strains, $\epsilon_{i j} \equiv \frac{1}{2}\left[\frac{\partial U_{i}}{\partial X_{j}}+\frac{\partial U_{j}}{\partial X_{i}}\right]$ and the stresses $\sigma_{i j}$ are defined in Love [11], and Boley and Weiner [12]. The cylinder is considered infinite to avoid end effects, therefore, the radial displacement, $U_{r}$, has no $z$-dependence. Since neither $\epsilon_{z z}$, nor $\epsilon_{r r}$, nor $\epsilon_{\theta \theta}$ depend on the $z$-variable, the differential equation is easily solvable for $f(r)$ :

$$
\frac{d^{2} f(r)}{d r^{2}}+\frac{1}{r} \frac{d f(r)}{d r}=0
$$

and leads to the following solution with $B_{0}$ and $r_{0}$ as integration constants.

$$
\begin{aligned}
f(r) & =B_{0} \ln \left(r_{0} / r\right) \\
U_{z} & =B_{0} z \ln \left(r_{0} / r\right) \\
\epsilon_{z z} & =B_{0} \ln \left(r_{0} / r\right) .
\end{aligned}
$$

The equilibrium equation in the radial direction may now be written for the region outside the cylinder:

$$
\frac{\partial \sigma_{r r}}{\partial r}+\frac{\partial \sigma_{r z}}{\partial z}+\frac{\sigma_{r r}-\sigma_{\theta \theta}}{r}=0
$$

with:

$$
\begin{aligned}
& \sigma_{r r}=(\lambda+2 \mu) \frac{\partial U_{r}}{\partial r}+\lambda \frac{U_{r}}{r}+\lambda \frac{\partial U_{z}}{\partial z} \\
& \sigma_{\theta \theta}=(\lambda+2 \mu) \frac{U_{r}}{r}+\lambda \frac{\partial U_{r}}{\partial r}+\lambda \frac{\partial U_{z}}{\partial z} \\
& \sigma_{r z}=-\mu B_{0} z / r .
\end{aligned}
$$

The resulting differential equation is again easily solvable: 


$$
\frac{d}{d r}\left[\frac{d U_{r}}{d r}+\frac{U_{r}}{r}\right]=\left(\frac{\lambda+\mu}{\lambda+2 \mu}\right) B_{0} / r
$$

with the following solution:

$$
U_{r}(r)=-\frac{B_{0}}{2}\left[\frac{\lambda+\mu}{\lambda+2 \mu}\right] r \ln \left(r_{0} / r\right)+Q / r .
$$

The characteristic length of the logarithmic term $r_{0}$, is taken to be the same as for $U_{z}$. While this assumption is made for simplicity in solving the problem, it is reasonable, as $r_{0}$ characterizes the penetration of the effect of the longitudinal stress into the matrix. $Q$ is a constant whose value will be determined by the boundary conditions. The logarithmic term in both solutions is only valid up to $r=r_{0}$. The following expressions are then obtained for the stresses outside the cylinder:

$$
\begin{gathered}
\sigma_{r r}=(\lambda+\mu) B_{0} / 2 \\
+\left[\lambda-\frac{(\lambda+\mu)^{2}}{(\lambda+2 \mu)}\right] B_{0} \ln \left(r_{0} / r\right)-2 \mu Q / r^{2} \\
\sigma_{\theta \theta}=\left[\lambda\left(\frac{\lambda+\mu}{\lambda+2 \mu}\right)\right] B_{0} / 2 \\
+\left[\lambda-\frac{(\lambda+\mu)^{2}}{(\lambda+2 \mu)}\right] B_{0} \ln \left(r_{0} / r\right)+2 \mu Q / r^{2} \\
\sigma_{z z}=\mu\left[\frac{3 \lambda+4 \mu}{\lambda+2 \mu}\right] B_{0} \ln \left(r_{0} / r\right)+\lambda\left[\frac{\lambda+\mu}{\lambda+2 \mu}\right] B_{0} / 2 .
\end{gathered}
$$

Inside the cylinder, the solutions resemble the "plane-strain" approximation with the exception of an allowed expansion of the cylinder in the longitudinal direction [see ref. [12], pp. 288-291].

$$
\begin{aligned}
& U_{r}^{\prime}(r)=\frac{1}{2}\left[\frac{3 \lambda+2 \mu}{\lambda+2 \mu}\right]\left[\frac{\Delta L}{L}\right] r \\
& U_{z}^{\prime}=A z
\end{aligned}
$$

$\frac{\Delta L}{L}$ is the total fractional expansion of the material in heating to the temperature of the track, and $A$ is a constant to be determined by the boundary conditions. Since the cylinder is small, we have not allowed a variation of $U_{z}$ with radial distance. This approximation proposes that the rod expands uniformly in the longitudinal direction, and greatly simplifies the problem without much effect on the final conclusions. The resulting stresses may be written as follows:

$$
\begin{aligned}
& \sigma_{r r}^{\prime}=\lambda A-\left[\frac{\mu(3 \lambda+2 \mu)}{(\lambda+2 \mu)}\right] \frac{\Delta L}{L} \\
& \sigma_{\theta \theta}^{\prime}=\lambda A-\left[\frac{\mu(3 \lambda+2 \mu)}{(\lambda+2 \mu)}\right] \frac{\Delta L}{L}
\end{aligned}
$$

$$
\begin{aligned}
& \sigma_{z z}^{\prime}=(\lambda+2 \mu) A-\left[\frac{2 \mu(3 \lambda+2 \mu)}{(\lambda+2 \mu)}\right] \frac{\Delta L}{L} \\
& \epsilon_{z z}^{\prime}=A .
\end{aligned}
$$

We now turn to boundary conditions to solve for the four unknown constants: $A, B_{0}, r_{0}$, and $Q$, assuming the following typical values for the Lamé constants and the calculated elongation, $\frac{\Delta L}{L}$ :

$$
\begin{aligned}
\lambda & =2.1 \times 10^{10} \mathrm{~N} / \mathrm{m}^{2} \\
\mu & =2.66 \times 10^{10} \mathrm{~N} / \mathrm{m}^{2} \\
\Delta L / L & =0.016 .
\end{aligned}
$$

The boundary conditions are as follows:

(i) the radial stress is continuous across the boundary:

$$
\sigma_{r r}(\mathrm{a})=\sigma_{r r}^{\prime}(\mathrm{a}) \quad \text { B.C. }-1
$$

(ii) the tangential strain and the radial displacement are continuous across the boundary:

$$
U_{r}(a)=U_{r}^{\prime}(a) \quad \text { B.C. }-2
$$

(iii) the longitudinal strain is continuous across the boundary:

$$
\epsilon_{z z}(\mathrm{a})=\epsilon_{z z}^{\prime}(\mathrm{a}) \quad \text { B.C. }-3
$$

(iv) there are no external longitudinal forces on the sample:

$$
\int_{S_{0}} \sigma_{z z} \cdot d s+\int_{S_{0}} \sigma_{z z}^{\prime} \cdot d s=0
$$

where $S_{0}$ is the cross-sectional area. This becomes:

$$
\int_{a}^{r_{0}} \sigma_{z z} r d r+\int_{0}^{a} \sigma_{z z}^{\prime} r d r=0 \quad \text { B.C. }-4
$$

Starting with B.C. -2 and B.C. -3 we calculate $Q$ from an estimate of $A$ :

$$
Q=2.82 \times 10^{-14}+7.23 \times 10^{-13} \mathrm{~A} .
$$

Then, $B_{0}$ and $r_{0}$ are calculated in turn from B.C. -1 and B.C. -3 as follows:

$$
\begin{gathered}
B_{0}=1.29 \mathrm{~A}+9.95 \times 10^{11} \mathrm{Q}-2.8 \times 10^{-2} \\
r_{0}=\operatorname{EXP}\left[\frac{A-13.41 B_{0}}{B_{0}}\right] .
\end{gathered}
$$

Finally, a new value of $A$ is computed from B.C. -4 and reinserted into B.C. -2 . The integrations of B.C. -4 for the region outside the cylinder are limited to $r_{0}$ 
since the solutions are only valid in this range. They lead to:

$$
A=10^{-2}+2.78 \times 10^{-5} B_{0}-1.23 \times 10^{12} B_{0} r_{0}^{2} .
$$

The iteration converges quickly and the following values are obtained for the constants, after we reached a variation smaller than 0.5 percent of each term:

$$
\begin{aligned}
& A=6.22 \times 10^{-4} \\
& Q=2.86 \times 10^{-14} \mathrm{~m}^{2} \\
& B_{0}=1.31 \times 10^{-3} \\
& r_{0}=2.46 \times 10^{-6} \mathrm{~m} .
\end{aligned}
$$

The stresses at the surface are then computed for $r=a=1.5 \mu \mathrm{m}$.

OUTSIDE ${ }^{3}$

$$
\begin{aligned}
& \sigma_{r r}=-6.5 \times 10^{8} \mathrm{~N} / \mathrm{m}^{2}=-95,000 \mathrm{psi} \\
& \sigma_{\theta \theta}=+6.9 \times 10^{8} \mathrm{~N} / \mathrm{m}^{2}=+100,000 \mathrm{psi} \\
& \sigma_{z z}=+4.6 \times 10^{7} \mathrm{~N} / \mathrm{m}^{2}=+6,800 \mathrm{psi}
\end{aligned}
$$

\section{INSIDE}

$$
\begin{aligned}
& \sigma_{z z}^{\prime}=-6.5 \times 10^{8} \mathrm{~N} / \mathrm{m}^{2}=-95,000 \mathrm{psi} \\
& \sigma^{\prime}{ }_{\theta \theta}=-6.5 \times 10^{8} \mathrm{~N} / \mathrm{m}^{8}=-95,000 \mathrm{psi} \\
& \sigma^{\prime}{ }_{z z}=-12.8 \times 10^{8} \mathrm{~N} / \mathrm{m}^{2}=-190,000 \mathrm{psi}
\end{aligned}
$$

Tensile stresses are positive while compressive ones are negative. Inside, the radial and tangential stresses are equal as in the plane strain problem, but their magnitude is slightly reduced due to the allowed longitudinal strain through the shear modulus. The longitudinal stress is seen to be very large, and all 3

\footnotetext{
${ }^{3} \mathrm{~A}$ shear stress, $\sigma_{r z}$ is also present outside. It reaches a large value near the ends of the tracks $\left(2 \times 10^{9} \mathrm{~N} / \mathrm{m}^{2}\right.$ for a track $0.2 \mathrm{~mm}$ long). However, since the tracks are finite, the glass matrix around the ends acts to dissipate the forces, and the model of an infinite track cannot matrix
apply.
}

stresses are compressive, as expected. Outside, the tangential and longitudinal stresses are tensile, with the former equaling three times the latter. The low value of the external longitudinal stress at the interface stems from the elastic distribution of the total force far into the matrix.

The authors wish to acknowledge the help of H. Dardy in producing the damage tracks and A. Napolitano and R. M. Waxler for measuring the high temperature refractive index and the thermal expansion of the undamaged base glass.

\section{References}

[1] Hercher, M., Laser-Induced Damage in Transparent Media, J. Opt. Soc. of America 54, 563 (1964).

[2] Davit, J., Filamentary damage in glasses (Proc. Symp. on Damage in Laser Materials, Boulder, Colorado, June 24-25, 1970), Nat. Bur. Stand. (U.S.), Spec. Publ. 341, 126 pages (Dec. 1970).

[3] Leitz Interference Microscope, Type SII, 50X-objective lens, 0.85 aperture.

[4] "Codalith," Kodak.

[5] Harper, D. W., Laser Damage in Glass, Brit. J. Appl. Phys. 16, 751-752 (1965).

[6] Ernsberger, F. M., Tensile and Compressive Strength of Pristine Glasses by an Oblate Bubble Technique, Physics and Chemistry of Glasses 10, 240-245 (1969).

[7] Ashkinadze, B. M., Valdimirov, V. I., Likhachev, V. A., Ryvkin, S. M., Salmanov, V. M., and Yardshetsyii, I. D., Damage Produced by Laser Beam in Transparent Dielectric, Soviet Physics-Doklady 11, No. 8, pp. 703-705 (Feb. 1967).

[8] Haller, W., and Dardy, H., Effect of microdomains formed by liquid/liquid immiscibility upon the damage threshold of glass, Nat. Bur. Stand. (U.S.), Tech. Note 574, p. 22 (May 1971).

[9] Carslaw, H. S., and Jaeger, J. C., Conduction of Heat in Solids, second edition (Clarendon Press, Oxford 1959).

[10] Simmons, J. H., and Macedo, P. B., Shear and Volume Structural Relaxation in Immiscible Oxide Melts, Amorphous Materials, R. W. Douglas and B. Ellis, Eds., Chapter 7 (John Wiley \& Sons, London 1971).

[11] Love, A. E. H., A Treatise on the Mathematical Theory of Elasticity, Fourth Edition (Dover, New York 1944).

[12] Boley, B. A., and Weiner, J. H., Theory of Thermal Stresses, Chapter 4 (John Wiley \& Sons, New York 1960).

[13] Wang, C. T., Applied Elasticity, Chapter 4 (McGraw-Hill, New York 1953).

(Paper 76A4-725) 